\title{
Using machine learning to characterize heart failure across the scales
}

\author{
M. Peirlinck • F. Sahli Costabal • K.L. Sack • J.S. Choy • G.S. Kassab • \\ J.M. Guccione · M. De Beule · P. Segers · E. Kuhl
}

Received: 6 March 2019 / Accepted: 16 June 2019

\begin{abstract}
Heart failure is a progressive chronic condition in which the heart undergoes detrimental changes in structure and function across multiple scales in time and space. Multiscale models of cardiac growth can provide a patient-specific window into the progression of heart failure and guide personalized treatment planning. Yet, the predictive potential of cardiac growth models remains poorly understood. Here we quantify predictive power of a stretch-driven growth model using a chronic porcine heart failure model, subject-specific multiscale simulation, and machine learning techniques. We combine hierarchical modeling, Bayesian inference, and Gaussian process regression to quantify the uncertainty of our experimental measurements during an eight-week long study of volume-overload in six pigs. We then propagate the experimental uncertainties from the organ scale through our computational growth model and quantify the agreement between experimentally measured and computationally predicted alterations on the cellular scale. Our study suggests that stretch is the major stimulus for myocyte lengthening
\end{abstract}

M. Peirlinck · M. De Beule · P. Segers

Biofluid, Tissue and Solid Mechanics for Medical Applications (IBiTech, bioMMeda), Ghent University, Ghent, Belgium

F. Sahli Costabal · E. Kuhl

Departments of Mechanical Engineering and Bioengineering, Stanford University, Stanford, California, USA

K.L. Sack

Department of Human Biology, University of Cape Town, Cape Town, South Africa

J.S. Choy · G.S. Kassab

California Medical Innovations Institute, Inc., San Diego, California, USA

K.L. Sack · J.M. Guccione

Department of Surgery, University of California at San Francisco, San Francisco, California, USA and demonstrates that a stretch-driven growth model alone can explain $52.7 \%$ of the observed changes in myocyte morphology. We anticipate that our approach will allow us to design, calibrate, and validate a new generation of multiscale cardiac growth models to explore the interplay of various subcellular, cellular, and organ level contributors to heart failure. Using machine learning in heart failure research has the potential to combine information from different sources, subjects, and scales to provide a more holistic picture of the failing heart and point towards new treatment strategies.

Keywords Machine learning - Gaussian process regression · Bayesian inference · uncertainty quantification · heart failure $\cdot$ growth and remodeling . multiscale

\section{Motivation}

Cardiovascular disease is the single leading cause of death worldwide with an estimated 17.3 million deaths in 2016, representing $31.5 \%$ of all global deaths [61]. With a 5-year mortality rate of $50 \%$, heart failure remains-and will remain in the foreseeable future-one of the most common, costly, disabling, and deadly medical conditions. In a traditional biomechanics sense, cardiac growth and remodeling, can be considered a protective and reparative homeostatic mechanism [20]. However, when combined with chronic alterations in the heart's loading conditions, these mechanisms lead to pathoanatomical changes that compromise the heart's electrical and mechanical function [52]. The underlying structural alternations can be categorized in two major patterns: eccentric hypertrophy associated with myocyte lengthening caused by a chronic overload in 

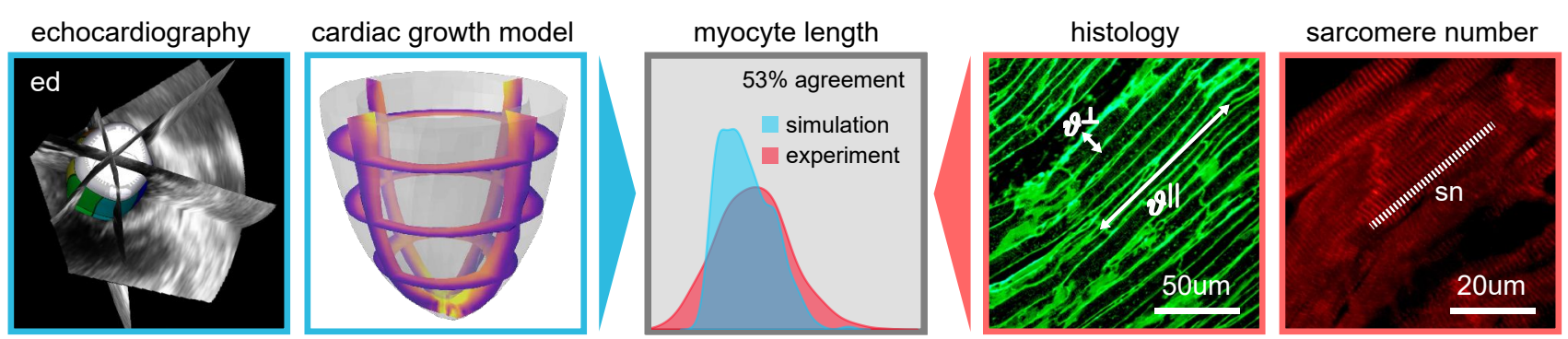

Fig. 1 Using machine learning and multiscale modeling to characterize heart failure across the scales. We characterize end diastolic and systolic volumes, myocyte widths and lengths, and sarcomere numbers and length in a longitudinal study of volume overload using cardiac echocardiography, tissue histology, and multiscale modeling. We quantify the agreement between simulation and experiment in terms of the myocyte lengths recorded in six animals throughout a period of eight weeks.

volume and concentric hypertrophy associated with myocyte thickening caused by a chronic overload in pressure [17]. Eccentric hypertrophy results in enlarged, dilated ventricles at a relatively constant wall thickness while concentric hypertrophy results in thickened ventricular walls at a relatively constant cardiac size [16]. In addition to a compromised mechanical function, these pathoanatomical changes distort the heart's electrical activation and conductivity resulting in an increasingly higher risk for ischemia, arrhythmia, and sudden death [25].

One of the most pertinent clinical questions in treatment planning is to anticipate and predict the rate of disease progression [65]. Consequently, the mechanobiological factors that regulate cardiac hypertrophy have been the subject of extensive research throughout the past decades [40]. Cardiac growth models have the potential to provide mechanistic insight in disease onset and progression [46] and can aid clinical decisionmaking or the design of emerging therapies [6]. Yet, a considerable debate remains around the underlying stimuli that trigger cardiac growth. Increasing evidence points to mechanical factors as mediating stimuli, both at the tissue and cellular levels. In eccentric hypertrophy, initial studies suggested that the end-diastolic stress would trigger cardiac dilation [19], while later studies opposed this hypothesis and suggested the enddiastolic stretch as a stimulus for cardiac growth [39]. Here we explore this hypothesis and characterize to which extent a mechanistic stretch-driven growth model can explain cardiac dilation in a unique chronic animal study where we induce and characterize eccentric hypertrophy across multiple lengths scales.

\section{Methods}

Figure 1 outlines the design of our study. To correlate whole organ, tissue, cellular, and subcellular lev- els, we use a multiscale continuum growth model. We characterize end diastolic volumes and myocyte dimensions in a longitudinal study of volume overload using echocardiography and tissue histology. To account for uncertainties in both sets of measurements, we combine Bayesian inference, uncertainty quantification, and Gaussian process regression. To quantify the predictive potential of our multiscale growth model, we characterize the agreement between the computationally simulated and experimentally measured myocyte lengths for six animals throughout a period of eight weeks.

\subsection{Animal model}

All animal experiments were performed at the California Medical Innovations Institute animal care facilities following an approved IACUC protocol according to national and local ethical guidelines, including the Guide for the Care and Use of Laboratory Animals, the Public Health Service Policy on Humane Care and Use of Laboratory Animals, and the Animal Welfare Act. As previously described [7,53], our study included six Yorkshire domestic pigs of either sex with a body weight of $46.3 \pm 8.0 \mathrm{~kg}$. At the day of surgery, the animals were sedated with telazol-ketamine-xylazine and anesthesia was maintained with isoflurane. To monitor the pressure and provide access to the left ventricle for mitral valve chordae disruption, we percutaneously inserted sheaths into the jugular vein to administer fluids and drugs into the right femoral artery. Using transesophageal echocardiography guidance, moderate to severe mitral regurgitation was created by disrupting one or more chordae. The degree of regurgitation was confirmed using echocardiography. At the day of surgery and then bi-weekly for a period of eight weeks, transthoracic and transesophageal echocardiograms were recorded and endomyocardial biopsy samples were collected. For the organ level characterization, 
we recorded echocardiographic images, two- and threedimensional in short and long axis views, with the animal in the supine and left lateral decubitus positions. From these images, we automatically constructed dynamic surface models of the left ventricle to extract end-diastolic volumes, end-systolic volumes, stroke volumes, ejection fractions, and wall thicknesses. For the cell level characterization, four to six endomyocardial biopsy samples were collected from the apex and the left ventricular free wall. We incubated the samples in cardioplegic solution at $37^{\circ} \mathrm{C}$, prepared 7 - $\mu$ m-thick slices, mounted them on glass slides, and incubated them with Wheat Germ Agglutinin to characterize myocyte widths and lengths and with Anti-Sarcomeric Alpha Actinin to characterize sarcomere numbers and lengths. Under fluorescence microscopy, the images were merged using ImageJ to identify the sarcomere numbers within each myocyte. At eight-weeks post surgery, the measurement protocol was terminated. At that time we deeply anesthetized the animal, arrested the heart in diastole with potassium chloride, and excised the heart. Finally, transmural punch biopsies were collected from the apex and free wall.

\subsection{Multiscale model of cardiac growth}

To correlate the changes in end diastolic volume to changes in myocyte length and width, we adopt the theory of finite growth [48]. This theory postulates a multiplicative decomposition of the deformation gradient $\boldsymbol{F}=\nabla \boldsymbol{\varphi}$, the gradient of the nonlinear deformation map $\boldsymbol{\varphi}$, into an elastic tensor $\boldsymbol{F}^{\mathrm{e}}$ and a growth tensor $\boldsymbol{F}^{\mathrm{g}}[1,30]$,

$\boldsymbol{F}=\nabla \boldsymbol{\varphi}=\boldsymbol{F}^{\mathrm{e}} \cdot \boldsymbol{F}^{\mathrm{g}}$

We assume that growth is transversely isotropic with respect to the myocyte direction $\boldsymbol{n}[37,66]$,

$\boldsymbol{F}^{\mathrm{g}}=\vartheta^{\|} \boldsymbol{n} \otimes \boldsymbol{n}+\vartheta^{\perp}[\boldsymbol{I}-\boldsymbol{n} \otimes \boldsymbol{n}]$,

and interpret the scalar longitudinal and transverse growth $\vartheta^{\|}$and $\vartheta^{\perp}$ as the relative changes in myocyte length and myocyte width $[38,64]$. Values of $\vartheta=1$ represent no change in myocyte dimensions, values of $\vartheta>1$ represent myocyte lengthening and thickening. Figure 2 illustrates the kinematics of our growth model, with the deformation $\boldsymbol{\varphi}$, the deformation gradient $\boldsymbol{F}$, the elastic tensor $\boldsymbol{F}^{\mathrm{e}}$, and the growth tensor $\boldsymbol{F}^{\mathrm{g}}$ as a direct result of the changes in myocyte length $\vartheta^{\|}$and width $\vartheta^{\perp}$. For the baseline elastic material response, we adopt the

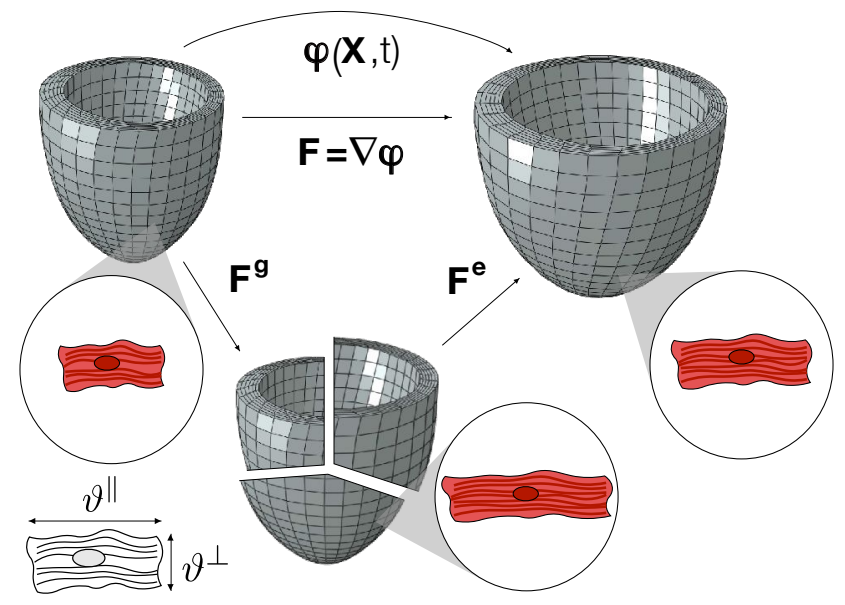

Fig. 2 Multiscale model of cardiac growth. Multiplicative decomposition of the deformation gradient $\boldsymbol{F}=\nabla \varphi=$ $\boldsymbol{F}^{\mathrm{e}} \cdot \boldsymbol{F}^{\mathrm{g}}$ into an elastic tensor $\boldsymbol{F}^{\mathrm{e}}$ and a growth tensor $\boldsymbol{F}^{\mathrm{g}}$; the growth tensor $\boldsymbol{F}^{\mathrm{g}}=\vartheta^{\|} \boldsymbol{n} \otimes \boldsymbol{n}+\vartheta^{\perp}[\boldsymbol{I}-\boldsymbol{n} \otimes \boldsymbol{n}]$ introduces the longitudinal and transverse growth $\vartheta^{\|}$and $\vartheta^{\perp}$, which are directly correlated to changes in myocyte length and width.

classical Holzapfel-Ogden model [22],

$$
\begin{aligned}
\psi & =\frac{a}{2 b} \exp \left(b\left[I_{1}^{\mathrm{e}}-3\right]\right)+\frac{a_{\mathrm{f}}}{2 b_{\mathrm{f}}}\left[\exp \left(b_{\mathrm{f}}\left[I_{4 \mathrm{f}}^{\mathrm{e}}-1\right]^{2}\right)-1\right] \\
& +\frac{a_{\mathrm{s}}}{2 b_{\mathrm{s}}}\left[\exp \left(b_{\mathrm{s}}\left[I_{4 \mathrm{~s}}^{\mathrm{e}}-1\right]^{2}\right)-1\right]+\frac{a_{\mathrm{fs}}}{2 b_{\mathrm{fs}}}\left[\exp \left(b_{\mathrm{fs}}\left(I_{8 \mathrm{fs}}^{\mathrm{e}}\right)^{2}\right)-1\right]
\end{aligned}
$$

where $I_{1}^{\mathrm{e}}, I_{4 \mathrm{f}}^{\mathrm{e}}, I_{4 \mathrm{~s}}^{\mathrm{e}}$, and $I_{8 \mathrm{fs}}^{\mathrm{e}}$ are the invariants that characterize the trace of the right elastic Cauchy Green deformation tensor, the elastic fiber stretch, sheet stretch, and coupled fiber-sheet stretch in terms of the elastic tensor $\boldsymbol{F}^{\mathrm{e}}$ [18] and $a, b, a_{\mathrm{f}}, b_{\mathrm{f}}, a_{\mathrm{s}}, b_{\mathrm{s}}, a_{\mathrm{fs}}$, and $b_{\mathrm{fs}}$ are the eight Holzapfel-Ogden material parameters. For the volume overload growth response, we adopt an established stretch-driven growth model in the longitudinal direction and assume that there is no growth in the thickness direction $[16,45,65]$,

$\dot{\vartheta}^{\|}=\frac{1}{\tau}\left\langle\lambda^{\mathrm{e}}-\lambda^{\mathrm{crit}}\right\rangle \quad$ and $\quad \dot{\vartheta}^{\perp}=0$.

The Macauley brackets \langle\rangle activate longitudinal growth only if the elastic fiber stretch, $\lambda^{\mathrm{e}}=\left[\boldsymbol{n} \cdot \boldsymbol{F}^{\mathrm{et}} \cdot \boldsymbol{F}^{\mathrm{e}} \cdot \boldsymbol{n}\right]^{1 / 2}$, exceeds the homeostatic baseline stretch, $\lambda^{\text {crit }}$. Importantly, $\lambda^{\text {crit }}$ is not a free parameter; it is the regionally varying homeostatic baseline stretch at end diastole, that we determine from a baseline simulation prior to inducing volume overload [51]. For $\lambda^{\mathrm{e}} \leq \lambda^{\text {crit }}$, there is no growth; for $\lambda^{\mathrm{e}}>\lambda^{\text {crit }}$, the growth parameter $\tau$ controls the speed of growth. 


\subsection{Computational model}

We simulate volume-overload induced growth using the finite element software package Abaqus [3]. For each animal, we create a finite element model of the left ventricle using the subject-specific echocardiography images at the minimum cavity volume of the cardiac cycle and assume that this is the stress free state. Figure 2 shows a typical left ventricular model discretized with 3,456 linear hexahedral elements, 4,152 nodes, and 12,456 degrees of freedom. We assign each element a discrete fiber orientation $\boldsymbol{n}$ using a rule-based approach [34] in which the fiber angles vary from $+60^{\circ}$ in the endocardium to $-60^{\circ}$ in epicardium [62]. We load the heart in two steps, homeostatic loading and pathologic overloading. For homeostatic loading, we load the ventricle to the experimentally recorded end diastolic volume and record and store the maximum stretch at each integration point as the critical stretch $\lambda^{\text {crit }}$ beyond which we activate growth. For pathologic overloading, we gradually increase the end diastolic volume. This results in an increase in the elastic fiber stretch $\lambda^{\mathrm{e}}$, which activates growth and triggers an increase in myocyte length $\vartheta^{\|}$.

\subsection{Parameter identification of elastic parameters}

To calibrate the eight Holzapfel-Ogden material parameters for each animal, we adopt a new two-stage approach [50]. First, we characterize an initial set of Holzapfel-Ogden parameters $\bar{a}, \bar{b}, \bar{a}_{\mathrm{f}}, \bar{b}_{\mathrm{f}}, \bar{a}_{\mathrm{s}}, \bar{b}_{\mathrm{s}}, \bar{a}_{\mathrm{fs}}, \bar{b}_{\mathrm{fs}}$ that reproduces ex vivo triaxial shear tests of human myocardial tissue [59]. Then, we map this set of ex vivo parameters onto our in vivo stiffness parameters $a, a_{\mathrm{f}}$, $a_{\mathrm{s}}$, and $a_{\mathrm{fs}}$ and our in vivo exponential parameters $b$, $b_{\mathrm{f}}, b_{\mathrm{s}}$, and $b_{\mathrm{fs}}$ via two scaling coefficients $\alpha$ and $\beta$ as $a_{i}=\alpha \bar{a}_{i}$ and $b_{i}=\beta \bar{b}_{i}[50]$. To calibrate the scaling coefficients $\alpha$ and $\beta$, we minimize the error between the analytical Klotz curve of the experimentally recorded end-diastolic pressure-volume relation at baseline [28] and the computationally simulated diastolic pressurevolume relation using Abaqus [8] and a sequential least squares programming optimization algorithm [24]. For the resulting set of in vivo Holzapfel-Ogden material parameters, the recorded end diastolic pressure inflates the ventricle from the recorded minimal volume to the recorded end diastolic volume, at which we record and store the homeostatic baseline stretch $\lambda^{\text {crit }}$ at each integration point.

\subsection{Sensitivity analysis of growth speed}

To explore the effects of the kinetics of growth, we systematically vary the only free parameter in the growth equation (4), the growth parameter $\tau$. We explore a wide range of growth parameters, $\tau=$ $[0.03,0.10,0.33,1.00,3.33,10.00]$ week, and highlight their effect on the longitudinal growth $\vartheta^{\|}$, the longitudinal growth rate $\dot{\vartheta}^{\|}$, and the elastic stretch in the fiber direction $\lambda^{\mathrm{e}}$. From this sensitivity analysis, we rationalize a growth parameter of $\tau=0.1$ week, which we use for all further studies. This value ensures a sufficiently rapid linear growth response to hemodynamic alterations which matches the quasi-constant growth rates observed in other experimental studies [63]. Importantly, throughout the entire process, we do not introduce any cellular information from tissue histology to prescribe growth as we have done in a previous study [53]. Rather, we use this cellular information to objectively assess the predictive power of the growth law.

\subsection{Uncertainty quantification using Bayesian inference}

To characterize volume-overload-induced changes in our organ and cell level variables, we employ Bayesian inference [53]. For all recorded samples, we postulate that our measurements are drawn from distributions that evolve in time. To ensure that the end diastolic volumes, myocyte lengths, and myocyte widths take positive values at all times, we assume that they are log-normally distributed at any point in time [36]. We further postulate that the myocyte length and width are correlated through a bivariate log-normal likelihood,

$\left[\begin{array}{c}\log \left(y_{\mathrm{ml}}^{i, j}\right) \\ \log \left(y_{\mathrm{mw}}^{i, j}\right)\end{array}\right] \sim \mathcal{N}\left(\left[\begin{array}{c}\mu_{\mathrm{ml}}^{i}\left(t_{j}\right) \\ \mu_{\mathrm{mw}}^{i}\left(t_{j}\right)\end{array}\right], \boldsymbol{\Sigma}\right)$,

where $y_{\mathrm{ml}}^{i, j}$ and $y_{\mathrm{mw}}^{i, j}$ are the myocyte length and width measurements $j$ of subject $i, \mu_{\mathrm{ml}}^{i}\left(t_{j}\right)$ and $\mu_{\mathrm{mw}}^{i}\left(t_{j}\right)$ are the means of their distributions at the time of measurement $j$, and $\boldsymbol{\Sigma}$ is the covariance matrix between these quantities. For the end diastolic volume, we assume a simple log-normal likelihood,

$\log \left(y_{\text {ed }}^{i, j}\right) \sim \mathcal{N}\left(\mu_{\text {ed }}^{i}\left(t_{j}\right), \sigma_{\text {ed }}\right)$,

where $y_{\text {ed }}^{i, j}$ is the $j$ th end diastolic volume measurement for subject $i, \mu_{\mathrm{ed}}^{i}\left(t_{j}\right)$ is the mean of its distribution at the time of measurement $j$, and $\sigma_{\text {ed }}$ is the standard deviation. We propose a linear relationship between the mean and time,

$\mu_{\mathrm{k}}^{i}(t)=a_{\mathrm{k}}^{i}+b_{\mathrm{k}}^{i} t \quad$ with $\mathrm{k}=\{\mathrm{ed}, \mathrm{ml}, \mathrm{mw}\}$, 
where $a_{\mathrm{k}}^{i}$ and $b_{\mathrm{k}}^{i}$ represent the intercept and slope of the line $\mu_{\mathrm{k}}(t)$ for subject $i$ and $k$ represents the end diastolic volume (ed), the myocyte length (ml) and the myocyte width $(\mathrm{mw})$ respectively. To take into account the variability between subjects and, at the same time, take advantage of the entire acquired dataset, we postulate a hierarchical model [12]. Specifically, we assume that the different parameters of the model in equation (7) for each subject $i$ are drawn from normal distributions, $a_{\mathrm{k}}^{i} \sim \mathcal{N}\left(\mu_{\mathrm{k}}^{a}, \sigma_{\mathrm{k}}^{a}\right)$ and $b_{\mathrm{k}}^{i} \sim$ $\mathcal{N}\left(\mu_{\mathrm{k}}^{b}, \sigma_{\mathrm{k}}^{b}\right)$. Altogether, this approach introduces four hyper-parameters, $\mu_{\mathrm{k}}^{a}, \mu_{\mathrm{k}}^{b}, \sigma_{\mathrm{k}}^{a}$ and $\sigma_{\mathrm{k}}^{b}$, which control the variability between the intercept $a_{\mathrm{k}}^{i}$ and slope $b_{\mathrm{k}}^{i}$ for each subject $i$. We can now estimate the individual response of each subject by considering the totality of the cohort. To complete our statistical model in a Bayesian setting, we select weakly informative priors and select our hyper parameters as $\mu_{\mathrm{k}}^{a}, \mu_{\mathrm{k}}^{b} \sim$ $\mathcal{N}\left(0,100^{2}\right)$ and $\sigma_{\mathrm{k}}^{a}, \sigma_{\mathrm{k}}^{b} \sim \operatorname{Half-\operatorname {Cauchy}}(\gamma=10)[11]$, and our remaining parameters as $\sigma_{\mathrm{k}} \sim \operatorname{Half-Cauchy}(\gamma=$ $100)$ and $\boldsymbol{\Sigma} \sim \operatorname{LKJ}(\eta=2)$, where LKJ denotes the Lewandowski-Kurowicka-Joe distribution [35]. This distribution is particularly suitable for multi-variate normal co-variance matrices [55]. Finally, we compute posterior distributions for the parameters using Bayes' theorem. We perform statistical inference using a Hamiltonian Monte Carlo method implemented in PyMC3 [55]. We then compute new samples of our quantities of interest $y_{\mathrm{k}}$ from a predictive posterior distribution at different time points and illustrate the discrete measurement points, their medians, their 95\% confidence intervals, and their inferred probability density functions throughout our entire study interval of eight weeks.

\subsection{Uncertainty propagation using Gaussian process regression}

To correlate the organ and cellular levels, we propagate the uncertainty of our experimental measurements through our computational model [54]. To account for the uncertainty on the organ level, we propagate the volume overload uncertainty to the predicted longitudinal growth. For each subject, ex supra, we characterize the uncertainty on the end diastolic volume, $\exp \left(a_{\mathrm{ed}}^{i}\right) \exp \left(b_{\mathrm{ed}}^{i} t\right)$, through hierarchical modeling and Bayesian modeling in terms of the parameters $a_{\text {ed }}^{i}$ and $b_{\text {ed }}^{i}$. Specifically, we normalize the volume overload by its value $\exp \left(a_{\text {ed }}^{i}\right)$ at time $t=0$, such that ed $=\exp \left(b_{\text {ed }}^{i} t\right)$, and characterize it by the last 500 samples of $b_{\text {ed }}$ from the Hamiltonian Monte Carlo chain. However, instead of performing a single forward growth simulation for each of these 500 samples, we train a
Gaussian process regression $[43,44]$ to predict the median longitudinal growth $\tilde{\vartheta} \|=f(t$, ed $)$ as a function of the time $t$ and the normalized end diastolic volume ed. For each subject, in the expected range of uncertainty, we perform $n=20$ simulations for training and an additional $n=10$ simulations for validation [42]. Since the intermediate steps towards the final solution are also valid data points, this represents a total of 621 points for training and 311 points for validation for each subject. After training the Gaussian process regression on the training set, the root mean squared error in median growth on the validation set was less than $0.27 \%$ for all animals. Once validated, we use the Gaussian process regression $f$ to propagate the 500 samples of $b_{\text {ed }}$ that characterize the volume overload uncertainty through the multiscale growth model. From these 500 samples, we determine the median longitudinal growth at 31 time points between weeks 0 and 8 . In total, this approach systematically generates 15,500 values of $\tilde{\vartheta} \|$ for each subject.

To account for the uncertainty in our cellular measurements, we correlate the longitudinal growth, $\vartheta^{\|}=$ $\exp \left(b_{\mathrm{ml}} t\right)$, to the parameter $b_{\mathrm{ml}}$ from the experimentally measured histology in equation (7). Similar to the volume overload, we normalize the myocyte length, $\exp \left(a_{\mathrm{ml}}\right) \exp \left(b_{\mathrm{ml}} t\right)$, from equation (5) by its initial value $\exp \left(a_{\mathrm{ml}}\right)$ at time $t=0$ to obtain $\mathrm{ml}(t)=\exp \left(b_{\mathrm{ml}} t\right)$ [53]. To quantify the agreement between the simulated and experimental longitudinal growth $\vartheta^{\|}$, we compute the probability density functions of both distributions at week 8 and determine the overlap as the percentage agreement between both probability density functions.

\section{Results}

3.1 End diastolic volumes increase with volume overload

Table 1 summarizes our echocardiography measurements at baseline and throughout a bi-weekly follow up period of eight weeks of volume overload. Altogether, we collected $n=28$ measurements of end diastolic and end systolic volumes. The mean end diastolic and end systolic volumes increased from $67.9 \pm 20.6 \mathrm{ml}$ and $30.2 \pm 9.5 \mathrm{ml}$ at baseline to $100.2 \pm 17.9 \mathrm{ml}$ and $51.3 \pm 9.0 \mathrm{ml}$ at week eight. This defines a decrease in ejection fraction from $55.6 \pm 4.9 \%$ to $47.7 \pm 9.5 \%$. Figure 3 shows the end diastolic volumes in all six animals in response to eight weeks of ventricular volume overload. The gray cross markers represent the $\mathrm{n}=28$ individual measurements, the solid black lines represent their medians, and dashed black lines their 
Table 1 Changes in end diastolic volume ed, end systolic volume es, myocyte length $\mathbf{m l}$, myocyte width $\mathbf{m w}$, sarcomere number sn, and sarcomere length sl in response to left ventricular volume overload. Results are summarized as mean \pm standard deviation.

\begin{tabular}{|c|rrrrr|l|}
\hline & w0 & w2 & w4 & w6 & w8 & $\Delta$ /week \\
\hline \hline ed & 67.9 & 71.3 & 77.7 & 86.8 & 100.2 & $+5.95 \%$ \\
{$[\mathrm{ml}]$} & \pm 20.6 & \pm 11.0 & \pm 9.5 & \pm 13.4 & \pm 17.9 & \\
\hline $\mathbf{e s}$ & 30.2 & 34.4 & 40.1 & 44.6 & 51.3 & $+8.73 \%$ \\
{$[\mathrm{ml}]$} & \pm 9.5 & \pm 7.2 & \pm 7.8 & \pm 5.7 & \pm 9.0 & \\
\hline \hline $\mathbf{m l}$ & 85.45 & 84.77 & 86.85 & 84.38 & 107.75 & $+3.26 \%$ \\
{$[\mu \mathrm{m}]$} & \pm 22.30 & \pm 17.17 & \pm 18.45 & \pm 18.93 & \pm 26.57 & \\
\hline $\mathbf{m w}$ & 13.67 & 13.04 & 14.19 & 13.85 & 12.98 & $-0.63 \%$ \\
{$[\mu \mathrm{m}]$} & \pm 3.73 & \pm 3.20 & \pm 2.96 & \pm 2.92 & \pm 3.01 & \\
\hline \hline $\mathbf{s n}$ & 47.50 & 49.91 & 50.36 & 49.97 & 62.07 & $+3.83 \%$ \\
{$[-]$} & \pm 11.67 & $\pm 9.96 \pm 10.76$ & \pm 11.62 & \pm 16.35 & \\
\hline $\mathbf{s l}$ & 1.75 & 1.78 & 1.75 & 1.76 & 1.77 & $+0.14 \%$ \\
{$[\mu \mathrm{m}]$} & \pm 0.15 & \pm 0.13 & \pm 0.10 & \pm 0.10 & \pm 0.11 & \\
\hline
\end{tabular}

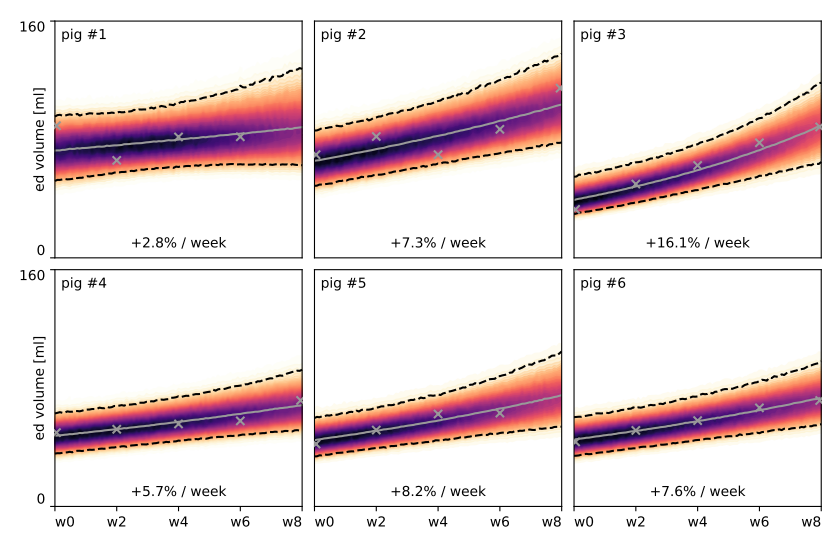

Fig. 3 Evolution of end diastolic volumes in response to left ventricular volume overload. Gray cross markers represent $n=28$ individual measurements of end diastolic volume, solid lines represent their medians, and dashed lines their $95 \%$ confidence intervals. Color contours from dark to light highlight the probability density from high to low.

95\% confidence intervals. The mean end diastolic volume increased by $+5.95 \% /$ week, with a rapid increase between weeks 6 and 8 . The direct comparison between the six animals illustrates the inter-animal variation: Pig \#3 displayed the largest increase in end diastolic volume with $+12.4 \% /$ week, pig \#1 the smallest with $+2.5 \% /$ week. Since the end systolic volume increased by $+8.73 \%$ /week, more rapidly than the end diastolic volume, the overall ejection fraction decreased, by $-1.78 \% /$ week. As the hearts dilated, the left ventricular mass increased by $9.88 \%$ /week.

\subsection{Myocyte lengths increase with volume overload}

Next to the echocardiography measurements, Table 1 also summarizes the histological measurements at

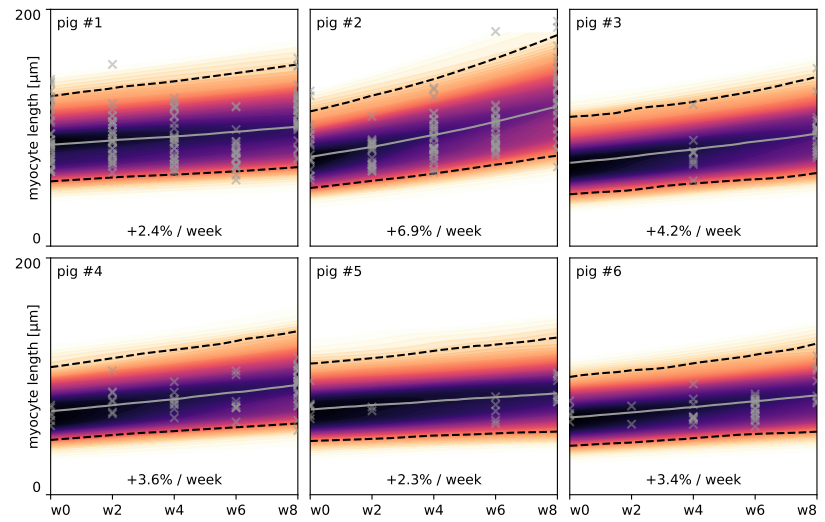

Fig. 4 Evolution of myocyte lengths in response to left ventricular volume overload. Gray cross markers represent $\mathrm{n}=460$ individual measurements of myocyte lengths, solid black lines represent their medians, and dashed black lines their $95 \%$ confidence intervals. Color contours from dark to light highlight the probability density from high to low.

baseline and throughout a bi-weekly follow up period of eight weeks of volume overload. Altogether, $\mathrm{n}=460$ measurements of myocyte length and width were collected. The mean myocyte length increased, from $85.45 \pm 22.30 \mu \mathrm{m}$ to $107.75 \pm 26.57 \mu \mathrm{m}$, with a relatively constant myocyte length throughout the first six weeks and a steep increase between weeks 6 and 8 . At the same time, the mean myocyte width remained virtually constant at $13.55 \mu \mathrm{m}$, varying marginally between minimum values of $12.98 \pm 3.01 \mu \mathrm{m}$ and maximum values of $14.19 \pm 2.92 \mu \mathrm{m}$. Figure 4 illustrates the myocyte length in all six animals in response to eight weeks of ventricular volume overload. The gray cross markers represent the $\mathrm{n}=460$ individual measurements, the solid black lines represent their medians, and dashed black lines their $95 \%$ confidence intervals. The mean myocyte length in Figure 4 increased markedly, by $+3.26 \% /$ week, with a rapid increase between weeks 6 and 8 . The direct comparison between the six animals illustrates the inter-animal variation: Pig \#2 displayed the largest increase in myocyte length with $+6.9 \%$ /week, pig \#5 the smallest with $+2.2 \%$ /week. Figure 5 illustrates the myocyte widths in all six animals in response to eight weeks of ventricular volume overload. The gray cross markers represent the $n=460$ individual measurements, the solid black lines represent their medians, and dashed black lines their 95\% confidence intervals. The mean myocyte width in Figure 5 varied only marginally, by $-0.63 \%$ /week. Pig \#2 displayed the largest increase in myocyte width with $+0.7 \%$ week, whereas pig \#6 showed the largest decrease with $-0.9 \% /$ week. This marginal change in myocyte width justifies the assumption of a constant my- 


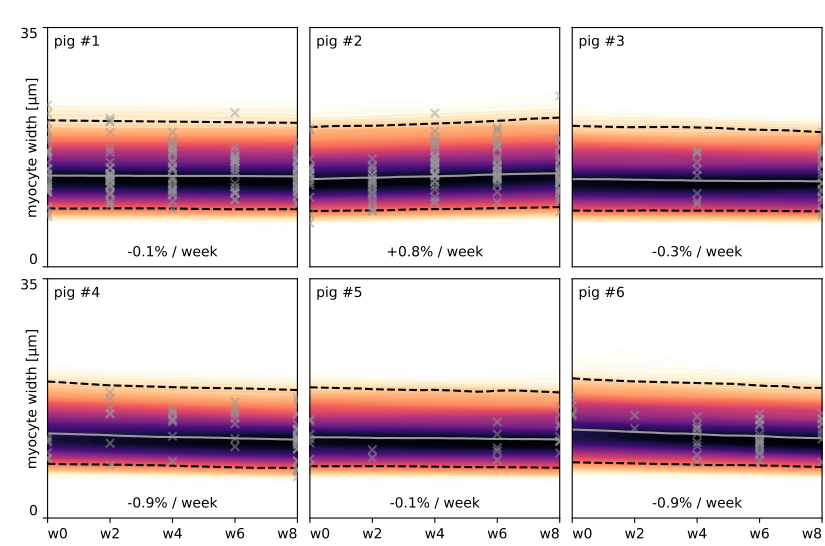

Fig. 5 Evolution of myocyte widths in response to left ventricular volume overload. Gray cross markers represent $\mathrm{n}=460$ individual measurements of myocyte widths, solid black lines represent their medians, and dashed black lines their 95\% confidence intervals. Color contours from dark to light highlight the probability density from high to low.

ocyte width $\vartheta^{\perp}=1$ and $\dot{\vartheta}^{\perp}=0$ in equation (4) of our growth model.

\subsection{Elastic behavior in vivo is softer than ex vivo}

Figure 6 depicts the fitted diastolic filling response for each pig following the subject-specific two-stage passive myocardial tissue reponse calibration. Table 2 summarizes our in vivo Holzapfel-Ogden material parameters for each animal in comparison to ex vivo human
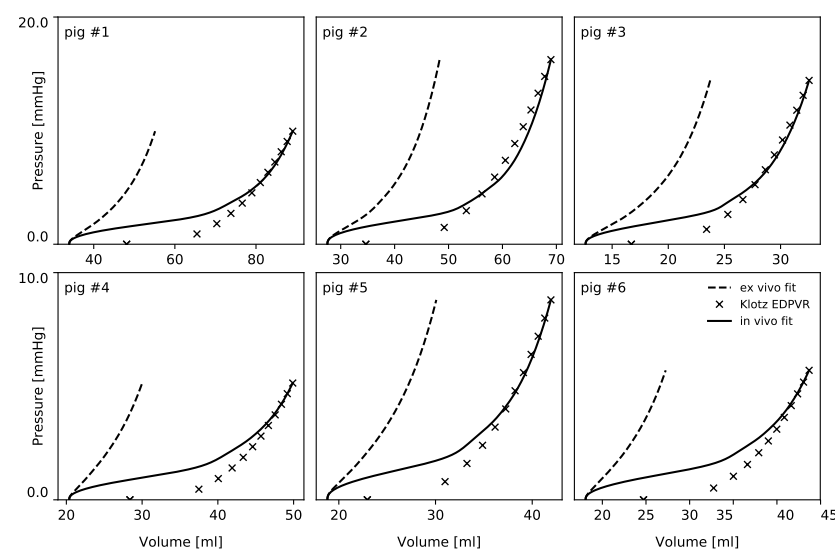

Fig. 6 Two-stage passive constitutive myocardial tissue response calibration. Dashed lines represent the diastolic filling pressure-volume relation based on ex vivo human Holzapfel-Ogden material parameters from the literature [50, 59], cross markers depict the theoretical end diastolic pressure volume relation (Klotz-curve) deduced from the experimentally measured subject-specific end diastolic pressure volume data [28] and solid lines depict the passive material response fitted to represent the subject-specific passive in vivo response.

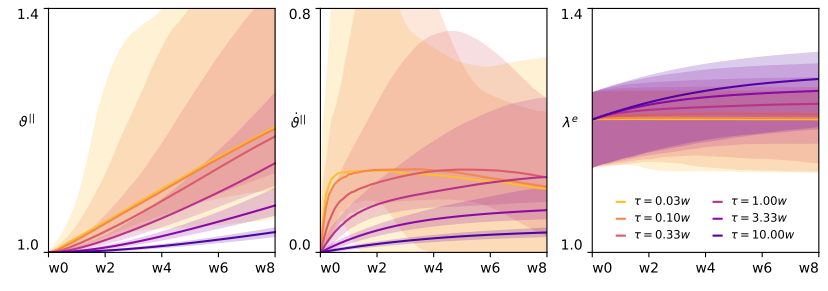

Fig. 7 Sensitivity analysis of growth with respect to the growth speed $\tau$. Longitudinal growth $\vartheta^{\|}$, longitudinal growth rate $\dot{\vartheta} \|$, and elastic stretch in the fiber direction $\lambda^{\mathrm{e}}$ averaged over the ventricle for varying growth speeds $\tau$, here illustrated for pig \#4. Solid lines represent the medians and shaded regions their $95 \%$ confidence intervals across the ventricle.

Holzapfel-Ogden material parameters from the literature $[50,59]$. We consistently identified stiffness parameters of the same order of magnitude, with a mean isotropic stiffness of $a=153.3 \pm 41.5 \mathrm{~Pa}$, and mean fiber and sheet stiffnesses of $a_{\mathrm{f}}=505.4 \pm 136.9 \mathrm{~Pa}$, $a_{\mathrm{s}}=70.2 \pm 19.0 \mathrm{~Pa}$, and $a_{\mathrm{fs}}=41.2 \pm 11.2 \mathrm{~Pa}$. The exponential parameters varied from $b=4.97 \pm 0.71$ for the isotropic term to $b_{\mathrm{f}}=9.54 \pm 1.36, b_{\mathrm{s}}=8.27 \pm 1.18$, and $b_{\mathrm{fs}}=2.04 \pm 0.29$ for the fiber and sheet terms. Notably, the stiffness parameters $a_{i}=\alpha \bar{a}_{i}$ took values on the order of $\alpha=14.60 \pm 3.95 \%$ of their ex vivo human counterparts $\bar{a}$. The exponential parameters $b_{i}=\beta \bar{b}_{i}$ were also smaller than their ex vivo human counterparts $\bar{b}$, but only by $\beta=65.93 \pm 9.40 \%$. The standard deviations in the two scaling parameters were small, $27.05 \%$ and $14.26 \%$ of their means, suggesting that our inversely identified in vivo stiffness parameters are consistently smaller than their ex vivo counterparts recorded [59] and identified [50] in the literature.

\subsection{Growth displays temporal and regional variations}

Figure 7 illustrates the results of our sensitivity analysis with respect to the growth speed $\tau$. The three graphs show the longitudinal growth $\vartheta^{\|}$, the longitudinal growth rate $\dot{\vartheta}^{\|}$, and elastic stretch in the fiber direction $\lambda^{\mathrm{e}}$ for varying growth speeds $\tau$ evaluated for pig \#4. The solid lines represent the medians and shaded regions their 95\% confidence intervals. According to equation (4), the parameter $\tau$ is inversely proportional to the longitudinal growth rate, $\dot{\vartheta}^{\|} \sim 1 / \tau$, as long as the elastic stretch $\lambda^{\mathrm{e}}$ exceeds the homeostatic baseline stretch $\lambda^{\text {crit }}$. In the limit of $\tau \rightarrow 0$, growth would take place instantaneously and the elastic stretch would immediately relax to the homeostatic baseline stretch, $\lambda^{\mathrm{e}}=\lambda^{\text {crit }}$. In the limit of $\tau \rightarrow \infty$, there would be no growth and the elastic stretch would always remain equal to the total stretch $\lambda^{\mathrm{e}}=\lambda$. Figure 7 shows that 
Table 2 In vivo Holzapfel-Ogden parameters identified individually for each animal at baseline compared to ex vivo HolzapfelOgden parameters in humans. Results are summarized as mean \pm standard deviation; $\alpha$ and $\beta$ are the animal-specific scaling factors of the stiffnesses $a_{i}$ and exponential parameters $b_{i}$ from ex vivo human to in vivo pig.

\begin{tabular}{|c||rr||rrrrrrrr|}
\hline ex vivo & $\begin{array}{r}\alpha \\
{[\%]}\end{array}$ & $\begin{array}{r}\beta \\
{[\%]}\end{array}$ & $\begin{array}{r}\bar{a} \\
{[\mathrm{~Pa}]}\end{array}$ & $\begin{array}{r}\bar{b} \\
{[-]}\end{array}$ & $\begin{array}{r}\bar{a}_{\mathrm{f}} \\
{[\mathrm{Pa}]}\end{array}$ & $\begin{array}{r}\bar{b}_{\mathrm{f}} \\
{[-]}\end{array}$ & $\begin{array}{r}\bar{a}_{\mathrm{s}} \\
{[\mathrm{Pa}]}\end{array}$ & $\begin{array}{r}\bar{b}_{\mathrm{s}} \\
{[-]}\end{array}$ & $\begin{array}{r}\bar{a}_{\mathrm{fs}} \\
{[\mathrm{Pa}]}\end{array}$ & $\begin{array}{r}\bar{b}_{\mathrm{fs}} \\
{[-]}\end{array}$ \\
\hline \hline human & 100.0 & 100.0 & 1050.0 & 7.54 & 3465.0 & 14.47 & 481.0 & 12.55 & 283.0 & 3.09 \\
\hline \hline in vivo & $\alpha$ & $\beta$ & $a$ & $b$ & $a_{\mathrm{f}}$ & $b_{\mathrm{f}}$ & $a_{\mathrm{s}}$ & $b_{\mathrm{s}}$ & $a_{\mathrm{fs}}$ & $b_{\mathrm{fs}}$ \\
& {$[\%]$} & {$[\%]$} & {$[\mathrm{Pa}]$} & {$[-]$} & {$[\mathrm{Pa}]$} & {$[-]$} & {$[\mathrm{Pa}]$} & {$[-]$} & {$[\mathrm{Pa}]$} & {$[-]$} \\
\hline \hline pig \#1 & 11.30 & 57.66 & 118.6 & 4.35 & 391.1 & 8.34 & 54.3 & 7.23 & 31.9 & 1.78 \\
pig \#2 & 15.62 & 68.64 & 164.0 & 5.18 & 540.6 & 9.93 & 75.1 & 8.61 & 44.1 & 2.12 \\
pig \#3 & 20.74 & 76.64 & 217.7 & 5.78 & 717.7 & 11.09 & 99.7 & 9.61 & 58.6 & 2.37 \\
pig \#4 & 10.01 & 57.36 & 105.1 & 4.32 & 346.5 & 8.30 & 48.1 & 7.20 & 28.3 & 1.77 \\
pig \#5 & 16.88 & 76.93 & 177.2 & 5.80 & 584.2 & 11.13 & 81.1 & 9.65 & 47.7 & 2.38 \\
pig \#6 & 13.07 & 58.37 & 137.2 & 4.40 & 452.2 & 8.45 & 62.8 & 7.32 & 36.9 & 1.80 \\
\hline \hline mean & 14.60 & 65.93 & 153.3 & 4.97 & 505.4 & 9.54 & 70.2 & 8.27 & 41.2 & 2.04 \\
std & \pm 3.95 & \pm 9.40 & \pm 41.5 & \pm 0.71 & \pm 136.9 & \pm 1.36 & \pm 19.0 & \pm 1.18 & \pm 11.2 & \pm 0.29 \\
\hline
\end{tabular}

for $\tau=0.03$ week and $\tau=0.10$ week, the response is close to the first limit case, with a linear longitudinal growth and a continuous relaxation of the elastic stretch to the homeostatic baseline stretch, $\lambda^{\mathrm{e}}=\lambda^{\text {crit }}$. In other words, for $\tau=0.10$ week, the growth model responds fast enough to always accommodate the echocardiographically measured increase in end diastolic volume. For values larger than $\tau=0.10$ week, the growth model responds slower than the measured increase in volume, and elastic stretches $\lambda^{\mathrm{e}}>\lambda^{\text {crit }}$ build up in the heart. Given that $\tau=0.10$ week results in a fairly constant growth rate $\dot{\vartheta}^{\|}$, which is in correspondence with other experimental volumetric overload studies [63], we selected $\tau=0.10$ week for all future simulations. As such, growth compensates the macroscopically observed changes in cardiac dimensions within a proper time frame, without building up a 'growth lag'. As this study set out to objectively assess the predictive power of an established growth law, we did not tune the growth speed parameter $\tau$ to the experimental data specifically. Figure 8 highlights the regional, transmural, temporal, and inter-animal variations of growth throughout our study. The volume overload of $12.4 \%$ /week for pig \# 3 , see Figure 3, triggered the largest longitudinal myocyte growth of up to $88 \%$; the volume overload of $2.5 \%$ /week for pig \# 1, see Figure 3, triggered the smallest growth of up to $31 \%$. Within each heart, growth displays notable transmural variations with larger values in the endocardium, the inner wall, and smaller values in the epicardium, the outer wall.

\subsection{Growth model correctly predicts myocyte} lengthening

For each animal, we successfully propagated the uncertainties of $\mathrm{n}=500$ end diastolic volumes from our

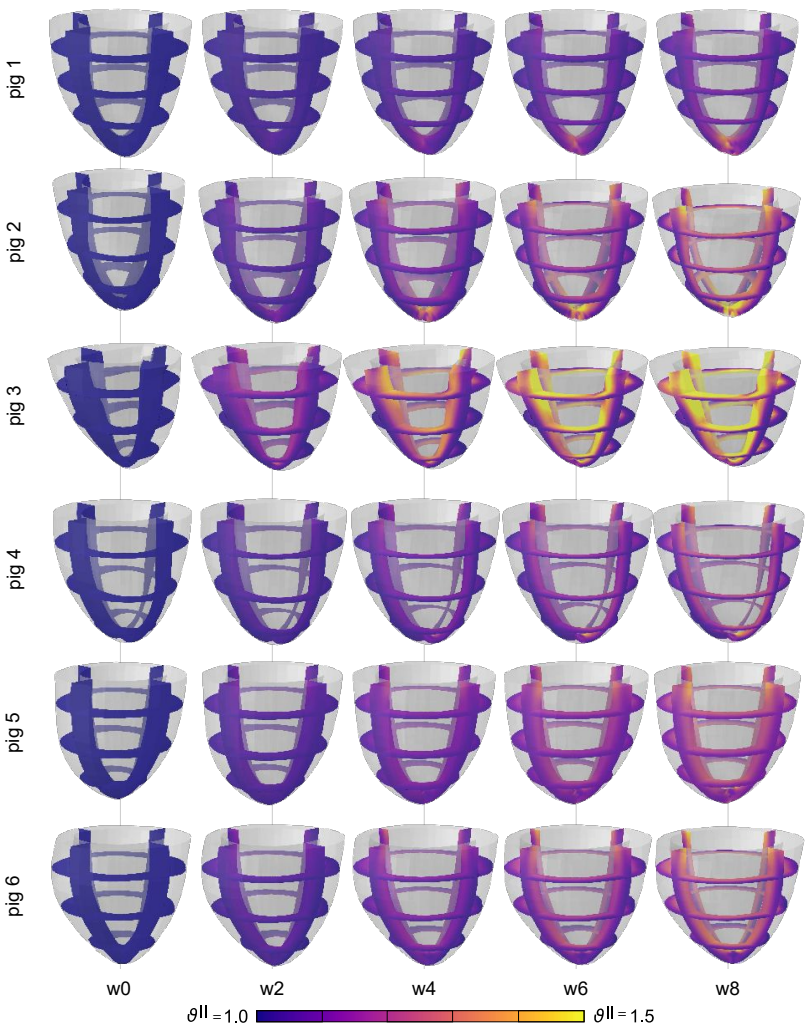

Fig. 8 Regional, transmural, temporal, and interanimal variations of growth in response to left ventricular overload. Longitudinal growth $\vartheta \|$ displays notable transmural variations with larger values in the endocardium, the inner wall, and smaller values in the epicardium, the outer wall. Growth is largest in pig \#3 and smallest in pig \#1.

echocardiography through the Gaussian process regression and obtained simulated myocyte morphologies, which we compared against our experimentally measured myocyte morphologies from our histology. Figure 9 compares our computationally simulated myocyte lengths across the entire left ventricular wall from the 


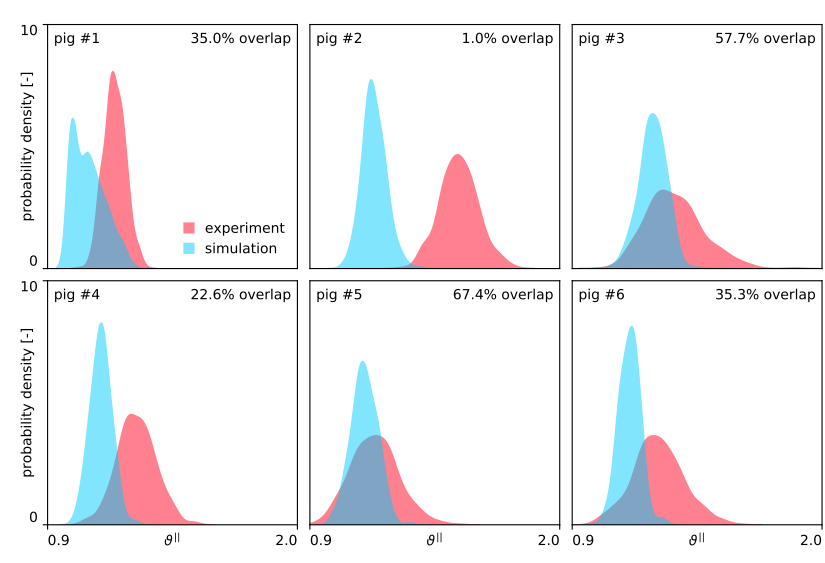

Fig. 9 Simulation and experiment of chronic myocyte lengthening across the entire ventricular wall. Blue and red probability density functions represent computationally simulated and experimentally measured myocyte lengths throughout the entire left ventricular wall, both with uncertainty; percentage agreement refers to the region shared between both graphs. The average agreement between simulation and experiment is $36.5 \%$.

Gaussian process regression to our experimentally measured myocyte lengths. The blue and red probability density functions represent simulation and experiment. The probability density functions are a qualitative and quantitative illustration of the intersubject variability of cardiac growth. The overlap between the blue and red graphs is a quantitative measure of the agreement between the model prediction and the experiment [23]. The average agreement between simulation and experiment is $36.5 \%$, ranging from a good agreement of $67.4 \%$ for pig \#5 to a poor agreement of $1.0 \%$ for pig \#2. For all six animals, the blue graphs are located to the left of the red graphs, which implies that the simulation underestimates the degree of myocyte lengthening. Since our histological samples originate exclusively from endocardial biopsies sampled at the inner wall, we now perform the same analysis, but this time by only using simulated myocyte lengths from the endocardial wall to more closely represent our experimental setting. Figure 10 compares our simulation of myocyte lengths across the endocardial wall from the Gaussian process regression to our experiment of histologically measured myocyte lengths. The experimental data are the same as in Figure 9, but now, the simulation only uses the myocyte lengths in the endocardial wall (inner 10\% of the ventricular wall thickness). Similar to Figure 9, the overlap between the blue and red graphs is a quantitative measure of the agreement between the model prediction and the experiment. The average agreement between simulation and experiment is $52.7 \%$, ranging from an excellent agreement of $85.7 \%$ for pig \#5 to a poor agreement of $6.0 \%$ for pig \#2. For all six an-

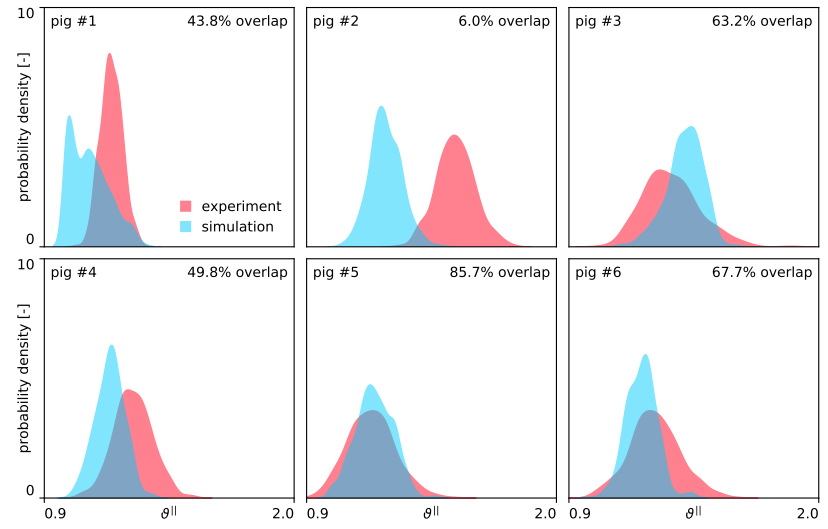

Fig. 10 Simulation and experiment of chronic myocyte lengthening across the endocardial wall. Blue and red probability density functions represent computationally simulated and experimentally measured myocyte lengths throughout the endocardial left ventricular wall, both with uncertainty; percentage agreement refers to the region shared between both graphs. The average agreement between simulation and experiment is $52.7 \%$.

imals, the blue graphs have now moved towards the right and are closer to the red graphs than in Figure 9 . This is in agreement with the regional variation of growth in Figure 8, which suggests that myocyte lengthening is larger in the endocardium, where the samples were taken, than in the epicardium. If we exclude pig $\# 2$, the largest pig of our study, from our analysis, the agreement for the remaining five pigs is $62.0 \%$. In other words, for pigs $\# 1, \# 3, \# 4, \# 5$, and \#6, the stretchdriven growth law coupled to alterations in end diastolic volume alone explain changes in myocyte length by $62.0 \%$. Taken together, Figure 10 suggests that elevated fiber stretches induced by an increase in diastolic volume are the major stimulus of chronic myocyte lengthening. Table 3 and Figure 11 compare the temporal evolution of the computationally simulated and experimentally measured changes in myocyte length. The simulated mean myocyte length increases by $2.84 \%$ per week, compared to the measured increase of $3.82 \%$ per week. This results in a simulated volume increase of $22.7 \%$ throughout the period of eight weeks compared to the experimentally measured increase of $30.6 \%$. Pig \#5 displays the best agreement with a simulated and measured weekly increase of $2.47 \%$ and $2.27 \%$ and an almost perfect overlap in Figure 11; pig \#2 displayed the least agreement with $3.05 \%$ and $6.92 \%$ and virtually no overlap in Figure 11.

\section{Discussion}

Cardiac growth is a complex process that affects the heart across multiple time and length scales. Accurate 


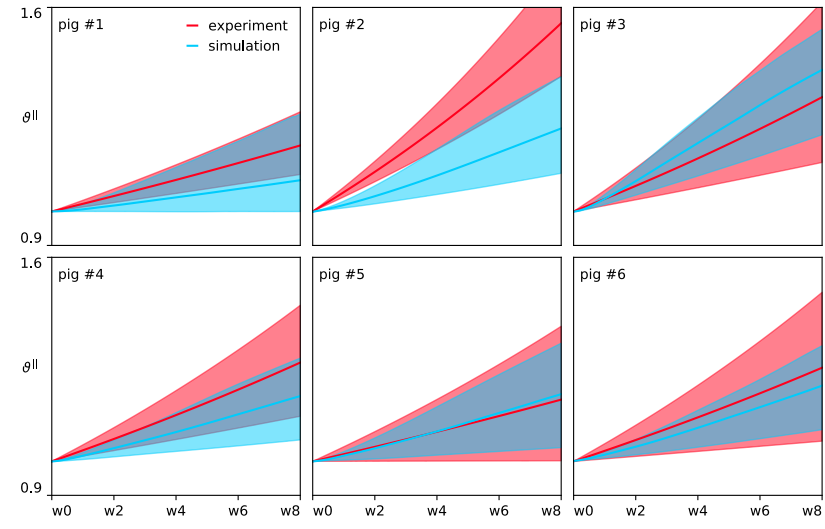

Fig. 11 Simulation and experiment of chronic myocyte lengthening across the endocardial wall. Blue lines represent medians and shaded regions $95 \%$ confidence intervals of computationally simulated myocyte lengthening in the endocardial wall; red lines represent medians and shaded regions $95 \%$ confidence intervals of experimentally measured myocyte lengthening from endomyocardial biopsies, both with uncertainties.

Table 3 Simulation and experiment of chronic myocyte lengthening across the endocardial wall. Computationally simulated myocyte lengths are recorded across the endocardial wall; experimentally measured myocyte lengths are determined from endomyocardial biopsies.

\begin{tabular}{|c|c|c|c|c|c|c|c|}
\hline pig & $\Delta \mathbf{m l}$ & w0 & w2 & w4 & w6 & w8 & $\Delta /$ week \\
\hline \multirow[t]{2}{*}{ \#1 } & $\operatorname{sim}$ & 1.00 & 1.02 & 1.04 & 1.07 & 1.09 & $+1.15 \%$ \\
\hline & $\exp$ & 1.00 & 1.05 & 1.09 & 1.14 & 1.19 & $+2.43 \%$ \\
\hline \multirow[t]{2}{*}{ \#2 } & $\operatorname{sim}$ & 1.00 & 1.04 & 1.11 & 1.17 & 1.24 & $+3.05 \%$ \\
\hline & $\exp$ & 1.00 & 1.12 & 1.25 & 1.39 & 1.55 & $+6.92 \%$ \\
\hline \multirow[t]{2}{*}{ \#3 } & $\operatorname{sim}$ & 1.00 & 1.09 & 1.20 & 1.31 & 1.42 & $+5.20 \%$ \\
\hline & $\exp$ & 1.00 & 1.08 & 1.16 & 1.24 & 1.34 & +4 \\
\hline \multirow[t]{2}{*}{ \#4 } & $\operatorname{sim}$ & 1.00 & 1.04 & 1.09 & 1.14 & 1.19 & $+2.40 \%$ \\
\hline & $\exp$ & 1.00 & 1.07 & 1.14 & 1.21 & 1.29 & $+3.63 \%$ \\
\hline \multirow[t]{2}{*}{ \#5 } & $\operatorname{sim}$ & 1.00 & 1.04 & 1.09 & 1.14 & 1.20 & $+2.47 \%$ \\
\hline & $\exp$ & 1.00 & 1.04 & 1.09 & 1.13 & 1.18 & $+2.27 \%$ \\
\hline \multirow[t]{2}{*}{ \#6 } & sim & 1.00 & 1.04 & 1.10 & 1.16 & 1.22 & $+2.78 \%$ \\
\hline & $\exp$ & 1.00 & 1.06 & 1.13 & 1.20 & 1.28 & $+3.44 \%$ \\
\hline \multirow[t]{2}{*}{$\overline{\text { overall }}$} & $\operatorname{sim}$ & $\bar{~} 1.00$ & 1.05 & $\overline{1.10}$ & 1.17 & $\overline{1.23}$ & $+2.84 \%$ \\
\hline & $\exp$ & 1.00 & 1.07 & 1.14 & 1.22 & 1.31 & $+3.82 \%$ \\
\hline
\end{tabular}

prediction of this process in response to exercise, disease, devices, or drugs could form an important clinical tool in designing new therapies for a range of pathological conditions. Recent reviews have begun to acknowledge the importance of multiscale computational modeling in providing additional insights into the pathological alterations of heart failure across the scales $[6,16$, $46,58]$. Following the very first theoretical mathematical approach of biological growth in the mid 90s [48], various groups have proposed different growth models for the heart throughout the past decade $[2,21,16,29$, $25,45,65]$. Motivated by numerous clinical [14,15,20, $40,56,58]$ and experimental $[32,57,60,63]$ studies that point to the importance of mechanical forces as modu- lators of cardiac growth, most of these growth models postulate mechanical measures as drivers for cardiac growth. It is clear from the wide variety of proposed growth laws that there still is considerable debate about the specific driving forces for growth [65]. Naturally, to identify the best basis for formulating growth models, it is critical to quantify the predictive power of a model in tight correlation with experimental data. However, such an assessment is challenging for multiple reasons: First, the highly patient-specific time course and extent of disease progression associated with hypertrophy calls for subject-specific data collection and modeling; second, the multiscale nature of cardiac growth requires the characterization at multiple spatial scales ranging from the subcellular via the cellular to the whole organ level; third, the intrinsic uncertainty associated with sparse experimental data has a strong influence on the predicted outcome.

Here we proposed a novel approach to quantify the predictive power of cardiac growth modeling using tools from machine learning. We designed a unique chronic volume overload study and monitored growth longitudinally, bi-weekly, across multiple spatial scales. We created subject-specific computational ventricle models, exposed them to subject-specific loading conditions, calibrated their subject-specific elastic material parameters under physiological conditions, and quantified the predictive power of a multiscale stretch-driven growth law under pathological conditions. Specifically, we performed a systematic sensitivity analysis to explore the role of the growth speed parameter, quantified uncertainty in experimental measurements using hierarchical modeling and Bayesian inference, and propagated the uncertainty through our subject-specific models using Gaussian process regression. In a previous purely kinematic study [53], we have used our experimental data to prescribe changes in cardiomyocyte dimensions and characterize resulting alterations in cardiac size. Now, we perform a dynamic study in which changes in cardiomyocyte dimensions evolve naturally as a consequence of the stretch-driven growth law. As such, this study truly probes the predictive potential of a multiscale growth model that correlates macroscopic changes in hemodynamic conditions to microscopic changes in myocardial architecture and myocyte ultrastructure. Importantly, nor the growth law, nor any growth parameter was tuned to lead to the best fit between simulated and experimental results. As such, we obtained an objective assessment of the growth law's predictive power in correlation with the experimental data. 
4.1 Ex vivo testing overestimates the elastic stiffness

Our study characterizes the individual subject-specific elastic response of myocardial tissue in the living heart using the Holzapfel-Ogden model [18,22]. Most existing cardiac growth models are not based on study- and subject-specific experimental data collected for such a purpose. Instead, they adopt elastic model parameters from previous studies designed for a different purpose [33]. The mismatch from using experimental data of other species or other studies, combined with the interanimal variability in elastic tissue behavior and left ventricular geometry, highlights the importance of subjectspecific modeling to accurately compute the regional stretch and stress distributions as potential drivers for cardiac growth. Specifically, here we adopt a new twostage method [50], which establishes ratios of material parameters using published ex vivo triaxial shear experiments [59] and maps the ex vivo parameters onto their subject-specific in vivo counterparts via two scaling coefficients using our own pressure-volume data [53] as showcased in Figure 6. The underlying assumption to reduce the set of unknowns is that the ratios between the four stiffness parameters $a, a_{\mathrm{f}}, a_{\mathrm{s}}$, and $a_{\mathrm{fs}}$ and the four exponential model parameters $b, b_{\mathrm{f}}, b_{\mathrm{s}}$, and $b_{\mathrm{fs}}$ remain unchanged in vivo and ex vivo, but their absolute values change linearly via the scaling coefficients $\alpha$ and $\beta$. Here, we identified the means of these coefficients to $\alpha=14.60 \%$ and $\beta=65.93 \%$. Table 2 reveals that the subject-specific scaling coefficients $\alpha$ and $\beta$ display a relatively low standard deviation of $27.05 \%$ and $14.26 \%$ amongst the six animals, while their means are significantly smaller than their ex vivo counterparts, here $85.40 \%$ and $34.07 \%$. This observation is significant since it suggests that the ex vivo identified HolzapfelOgden stiffness parameters [22] overestimate the myocardial stiffness by more than one order of magnitude. The drastic increase in myocardial stiffness from in vivo to ex vivo is a collective result of ultrastructural and architectural changes within the myocardial tissue post mortem and upon explantation. This trend is consistent with prior studies of $\alpha=16.0 \%$ and $\beta=73.0 \%$ for porcine [50] and $\alpha=8.98 \%$ and $\beta=77.9 \%$ for human [41] myocardial tissue in vivo. In summary, we suggest using Holzapfel-Ogden stiffnesses of $a=153.3$ $\mathrm{Pa}, a_{\mathrm{f}}=505.4 \mathrm{~Pa}, a_{\mathrm{s}}=70.2 \mathrm{~Pa}$, and $a_{\mathrm{fs}}=41.2 \mathrm{~Pa}$, and $b=4.97, b_{\mathrm{f}}=9.54, b_{\mathrm{s}}=8.27$, and $b_{\mathrm{fs}}=2.04$ when simulating porcine myocardial tissue in vivo. Since the standard deviations on these values were low-all less than 30\%-we can confidently recommend these values for future simulations without the need to personalize or re-identify these parameters again in future simulations.
4.2 Growth displays temporal and regional variations

To illustrate the temporal evolution of growth, we performed a systematic sensitivity analysis of the growth parameter $\tau$ in Figure 7. Our myocyte morphologies in Table 1 reveal that our mean myocyte lengthening of $+3.26 \%$ /week results in a total myocyte lengthening of $26.08 \%$ throughout the period of eight weeks, while the mean myocyte width remains virtually constant with a small reduction of $-0.63 \% /$ week. This agrees well with a human cardiomyopathy study in which myocyte lengths from freshly isolated cardiac tissue were on average $40 \%$ longer in the cardiomyopathy group than in the healthy control group, while the myocyte widths displayed no statistically significant differences between both groups [14]. Our histology explains chronic myocyte lengthening through an increase in the mean serial sarcomere number of $+3.83 \%$ /week resulting in a total increase in the serial sarcomere number of $30.64 \%$ within eight weeks, while the mean sarcomere length remains unchanged with a small increase of $+0.02 \%$ /week. A previous chronic volume overload study in rabbits has shown that myocytes compensate for chronic overstretch by adding sarcomeres in series, initially at a constant rate of approximately one sarcomere per day [63]. Our mean myocyte lengths also increased linearly in time, from 85.45 to 107.75 , by adding sarcomeres in series, from 47.50 to 62.07 , at a constant rate of one sarcomere per four days [53]. This agrees well with our sensitivity analysis in Figure 7, from which we have rationalized a growth parameter of $\tau=0.10$ weeks associated with a linear increase in myocyte length $\vartheta^{\|}$at a constant rate $\dot{\vartheta} \|$. Our four-fold slower sarcomere addition in pigs compared to rabbits [63] translates into a four-fold difference in the parameter $\tau$, and could be a natural consequence of the four-fold difference in metabolic rate between pigs and rabbits [27].

To illustrate the regional, transmural, and intra-animal variations of growth we plot the myocyte lengthening $\vartheta^{\|}$across the entire volume overloaded left ventricle in Figure 8. Our model reveals a pronounced heterogeneity in the cellular response to volume overload with myocytes in the endocardium, the inner wall, growing more than myocytes in the epicardium, the outer wall. These regional variations agree well with previous volume overload simulations in an idealized bi-ventricular model [17] and in a personalized human heart model [13], and with chronic volume overload experiments in sheep [60], where myocyte lengthening was more pronounced in the endocardial than in the epicardial wall. The observed regional, transmural, and inter-animal heterogeneity of growth elucidates the importance of 
subject-specific modeling of growth to avoid erroneous results and conclusions.

4.3 Bayesian inference reveals chronic dilation and myocyte lengthening

Our study characterizes volume overload induced growth at a uniquely high spatial and temporal resolution; however, it is still constrained by the natural limitations of a chronic large animal study associated with sparse and unevenly distributed data for each individual animal [53]. Using the concepts of hierarchical modeling and Bayesian inference proved crucial to interpret our experimental measurements in view of the total cohort of animals. For example, pig \#3 only has $\mathrm{n}=5$ myocyte morphology recordings at week 4 and $\mathrm{n}$ $=11$ at week 8 . Nonetheless, when interpreted within the total cohort of all six subjects, we can observe clear trends of pronounced myocyte lengthening at $+4.3 \%$ per week in Figure 4 and of marginal myocyte thinning of $-0.3 \%$ per week in Figure 5 . Using tools from machine learning to interpret our data also allows us to quantify the uncertainty of our experimental measurements and provides confidence in the finding that volume overload triggers chronic dilation and myocyte lengthening. On the whole organ level, our results in Table 1 and Figure 3 confirm the common understanding that a chronic volume overload induces an increase in end diastolic volume. This results in a decrease in ejection fraction, from $55.6 \%$ to $47.7 \%$, which agrees qualitatively with clinical observations [31], but is quantitatively less pronounced than in end-stage heart failure patients [14]. A mean weekly increase in end diastolic volume of $+5.95 \%$ results in a total increase of $47.6 \%$ after only eight weeks. These values are in good agreement with a clinical study where 88 hypertensive patients were compared to 29 healthy controls [47]. On the cellular level, Figure 4 reveals significant changes in myocyte length of $+3.83 \%$ per week, whereas Figure 5 suggests negligible changes in the myocyte width of $-0.63 \%$ per week. The myocyte lengthening and fairly constant myocyte width result in a myocyte length-to-width ratio of 6.3 at baseline and of 8.3 after eight weeks of volume overload. These results are in good agreement with the reported myocyte length-to-width ratios ranging from 7.0 in the healthy left ventricle [4] to 11.5 in the failing left ventricle [14]. Additionally, the approximately constant width of the confidence intervals in Figures 3, 4 and 5 confirms that the linear evolution assumption on the log-normal mean of the end diastolic volume, the myocyte length, and the myocyte width in equation (7) is appropriate to describe the temporal evolution of the collected experimental data. In summary, using tools from machine learning, hierarchical modeling, Bayesian inference, and uncertainty quantification, proved critical to interpret our unevenly distributed data and to quantify the intrinsic uncertainties in our experimental measurements. Using these methods in real time - in parallel to an ongoing study - could guide strategies of adaptive sampling, for example by purposely selecting non-equidistant, unevenly distributed time points to efficiently explore the parameter space and maximize the information content of each measurement [54].

\subsection{Uncertainty propagation provides quantitative} insight into the quality of our model

To date, there is no established method to bridge subcellular, cellular, tissue, and organ levels and to characterize how heart failure alters cardiac form and function across the scales. In a previous study, we have used a purely kinematic approach to connect the sarcomere, myocyte, myocardial, and whole heart levels [53]. Here we use a dynamic approach and connect the scales via a multiscale cardiac growth model to predict how myocyte morphologies evolve dynamically as a result of ventricular volume overload. Most importantly, we have designed an experimental study that collects data across the scales to precisely quantify the predictive features of our model. Specifically, our mechanistic multiscale growth model is sensitive to volumetric overload, which initially drives individual myocytes away from their homeostatic equilibrium state until they have deposited enough sarcomeres in series to regain homeostatic equilibrium. We quantify the uncertainties in our volume overload recordings that drive myocyte lengthening and propagated this uncertainty through our growth model $[43,44]$ to the predicted changes in myocyte morphology. However, rather than performing a computationally expensive growth simulation for each realization, we create a computationally efficient surrogate model from Gaussian process regression and propagate the uncertainties through this surrogate model [42]. Figure 9 provides a side-by-side comparison of the experimentally measured and the computationally predicted myocyte lengths after eight weeks, both with uncertainties. The mean overlap between both graphs defines the agreement of model and experiment of $36.5 \%$. Since the biweekly biopsy samples for our experimentally measured myocyte morphologies were collected on the endocardial surface of the left ventricle, we re-did the analysis, but now by only sampling our computationally simulated myocyte morphologies across the endocardial wall. Figure 10 shows the quantitative comparison between experimentally measured and computationally predicted myocyte lengths. The agreement 
ranges from excellent with $85.7 \%$ for pig \#5 to poor with $6.0 \%$ for pig \#2. On average, our growth model displays a predictive power of $52.7 \%$ to predict myocyte lengths from ventricular volume overload. The average agreement of $36.5 \%$ and $52.7 \%$ in Figures 9 and 10 is slightly lower than the average agreement of $54.0 \%$ in our previous, purely kinematic analysis [53]. This observation is not unexpected, since our predictive growth model of the dynamic evolution of myocyte lengths allows for more freedom than our previous, purely kinematic enforcement of the correlation between the scales. If we exclude pig \#2, the largest pig and a potential outliner, from this analysis, the predictive power increases to $62.0 \%$. Overall, this quantitative assessment of our model suggests that other contributing factors, e.g., myocardial disarray or extracellular matrix remodeling, could play an important role in heart failure and should be included and quantified in future model refinements.

\subsection{Limitations}

This study proposes a new strategy to develop, calibrate, and validate a multiscale growth model that tightly integrates experiment and simulation using tools from machine learning. While this allows us, for the first time, to quantify the predictive power of a stretch-based growth law, our study has a few limitations: First, in view of the relatively large inter-animal variability, our initial number of six subjects seems rather low. With our proposed methods, we can easily integrate new subjects into our study and explore the predictive power of our stretch-driven growth model for a larger group. This could also help us to better explain the discrepancies for pig \#2 and to assess the uncertainty on the growth rate parameter $\tau$. Second, our subjects were all monitored bi-weekly, throughout a limited time period of eight weeks. In retrospect, it would have been interesting to continue the study for a few more weeks to more closely approach end stage heart failure. If we had known upfront that we would use Bayesian inference to analyze our data, we could have designed the study more interactively and used adaptive sampling to more efficiently sample the parameter space. Third, we have selected a relatively simple growth model with only one free parameter, the parameter $\tau$. While we have rationalized this parameter from a systematic sensitivity analysis and other experimental studies, a follow-up study could try to 'learn' this parameter from the experimental data specifically. However, a longer study interval would be needed to truly identify the growth parameter $\tau$ and compare our simple stretch-driven model to more complex stretch- or stress-driven models for growth. For the considered stretch-driven growth law, a mere tuning of $\tau$ to the temporally changing histology data is not expected to improve the overlap. More specifically, the $\tau=0.10$ week rationalization renders nearly-maximal longitudinal growth for the considered growth law and still underestimates the myocyte lengthening at week 8 for pig \#1, \#2 and \#4. Fourth, we have induced left ventricular volume overload in pigs through controlled regurgitation to ultimately gain insight into the progression of heart failure in humans. While ventricular volumes and myocyte dimensions are relatively comparable between pigs and humans, it remains to be shown to which extent the timeline of cellular and ventricular adaptation to volume overloaded pigs translates into the timeline of heart failure in humans. Finally, when creating our subject-specific ventricle models at baseline, multiple modeling aspects including the constitutive material parameters, the ventricular geometry, the myofiber architecture, and the boundary conditions, can have a potential effect on the predicted ventricular mechanics $[5,10,41,49]$. Quantifying the effect of each of these modeling aspects is beyond the scope of this study. Instead, we followed the current best practice to create deterministic subject-specific ventricular models for each pig at baseline [50].

\section{Conclusion}

Multiscale cardiac growth models hold huge promise for simulating the patient-specific timeline of heart failure and for optimizing surgical or pharmacological treatment strategies. While scientists are increasingly recognizing the predictive potential of cardiac growth models, the clinical community is rather skeptical, mainly because the predictive power of these models remains unknown. Here we design a new approach to quantify the predictive power of cardiac growth models by combining multiscale experiments and simulations using tools of machine learning. We adopt techniques of hierarchical modeling, Bayesian inference, and Gaussian process regression to quantify the uncertainty of our experimental measurements and propagate this uncertainty through a surrogate model for cardiac growth to predict changes in myocyte morphology. Our results suggest that stretch-driven growth is the major contributor to alterations in myocyte morphology. Our simple growth model alone can explain $52.7 \%$ of the observed changes in myocyte length. Using machine learning in heart failure research allows us to seamlessly integrate experimental, computational, and clinical data from different scales and times and to design efficient experimental and clinical studies that adaptively sample the parameter space at varying points in space and 
time. Machine learning tools naturally provide highresolution quantitative insight into the quality of the experiments and the predictive potential of the models. Ultimately, our approach has the potential to combine information from different sources, subjects, and scales to provide a more holistic picture of the failing heart and point towards new treatment strategies.

Acknowledgements This work was supported by the Flanders Innovation and Entrepreneurship Agency (VLAIO) strategic basic research grant 141014 and a travel grant by the Flemish Fund for Scientific Research (FWO) to Mathias Peirlinck, by the Becas Chile-Fulbright Fellowship to Francisco Sahli Costabal, and by the National Institutes of Health Grant U01 HL119578 to Julius M. Guccione, Ghassan S. Kassab, and Ellen Kuhl.

\section{References}

1. D. Ambrosi, G.A. Ateshian, E.M. Arruda, S.C. Cowin, J. Dumais, A. Goriely, G.A. Holzapfel, J.D. Humphrey, R. Kemkemer, E. Kuhl, J.E. Olberding, L.A. Taber, K. Garikipati. Perspectives on biological growth and remodeling. J. Mech. Phys. Solids. 59 (2014) 863-883.

2. T. Arts, T. Delhaas, P. Bovendeerd, X. Verbeek, F.W. Prinzen. Adaptation to mechanical load determines shape and properties of heart and circulation: the CircAdapt model. Am. J. Phys. Heart Circ. Phys. 288 (2005) H1943-H1954

3. B. Baillargeon, N. Rebelo, D.D. Fox, R.L. Taylor, E. Kuhl. The Living Heart Project: A robust and integrative simulator for human heart function. Eur. J. Mech. A/Solids 48 (2014) 38-47.

4. M.A. Bray, S.P. Sheehy, K.K. Parker. Sarcomere alignment is regulated by myocyte shape. Cell Motil. Cytoskel. 65 (2008) 641-651

5. J.O. Campos, J. Sundnes, R.W. dos Santos, B.M. Rocha. Effects of left ventricle wall thickness uncertainties on cardiac mechanics. Biomech. Model. Mechanobiol. doi:10.1007/s10237-019-01153-1 (2019)

6. R. Chabiniok, V. Wang, M. Hadjicharalambous, L. Asner, J. Lee, M. Sermesant, E. Kuhl, A. Young, P. Moireau, M. Nash, D. Chapelle, D.A. Nordsletten. Multiphysics and multiscale modeling, data-model fusion and integration of organ physiology in the clinic: ventricular cardiac mechanics. Interface Focus 6 (2016) 20150083.

7. J.S. Choy, S. Leng, Y. Awakeem, K.L. Sack, Y. Dabiri, L. Zhong, J.M. Guccione, G.S. Kassab. Mechanical stretch as stimulus for growth and remodeling in mitral regurgitation. submitted for publication.

8. Dassault Systèmes, SIMULIA. 2018. Abaqus 2018, Documentation. Dassault Systèmes, Rhode Island.

9. S. Dokos, B.H. Smaill, A.A. Young, I.J. LeGrice. Shear properties of passive ventricular myocardium. Am. J. Physiol. Heart Circ. Physiol. 283 (2002) H2650-H2659

10. T.S.E. Eriksson, A.J. Prassl, G. Plank, G.A. Holzapfel. Influence of myocardial fiber/sheet orientations on left ventricular mechanical contraction. Math. Mech. Solids 18 (2013) 592-606

11. A. Gelman. Prior distributions for variance parameters in hierarchical models. Bayesian Anal. 1 (2006) 515-534

12. A. Gelman, J. Hill. Data analysis using regression and multilevel/hierarchical models. Cambridge University Press, Cambridge, 2006
13. M. Genet, L.C. Lee, B. Baillargeon, J.M. Guccione, E. Kuhl. Modeling pathologies of systolic and diastolic heart failure. Ann. Biomed. Eng. 44 (2016) 112-127

14. A.M. Gerdes, S.E. Kellerman, J.A. Moore, K.E. Muffly, L.C. Clark, P.Y. Reaves, K.B. Malec, P.P. Mc Keown, D.D. Schocken. Structural remodeling of cardiac myocytes in patients with ischemic cardiomyopathy. Circulation 86 (1992) 426-430

15. A.M. Gerdes, J.M. Capasso. Structural remodeling and mechanical dysfunction of cardiac myocytes in heart failure. J. Mol. Cell Cardiol. 27 (1995) 849-856.

16. S. Göktepe, O.J. Abilez, E. Kuhl. A generic approach towards finite growth with examples of athlete's heart, cardiac dilation, and cardiac wall thickening. J. Mech. Phys. Solids 58 (2010) 1661-1680

17. S. Göktepe, O.J. Abilez, K.K. Parker, E. Kuhl. A multiscale model for eccentric and concentric cardiac growth through sarcomerogenesis. J. Theor. Bio. 265 (2010) 433442

18. S. Göktepe, S.N.S. Acharya, J. Wong, E. Kuhl. Computational modeling of passive myocardium. Int. J. Num. Meth. Biomed. Eng. 27 (2011) 1-12

19. W. Grossman, D. Jones, L.P. McLaurin. Wall stress and patterns of hypertrophy in the human left ventricle J. Clin. Invest. 56 (1975) 56-64

20. W. Grossman. Cardiac hypertrophy: useful adaptation or pathologic process? Am. J. Med. 69 (1980) 576-584

21. J.W. Holmes. Candidate mechanical stimuli for hypertrophy during volume overload. J. Appl. Physiol. 97 (2004) 1453-1460

22. G.A. Holzapfel, R.W. Ogden. Constitutive modelling of passive myocardium: a structurally based framework for material characterization. Philos. Trans. A. Math. Phys. Eng. Sci. 367 (2009) 3445-3475

23. H.F. Inman, E.L. Bradley Jr, The overlapping coefficient as a measure of agreement between probability distributions and point estimation of the overlap of two normal densities. Comm. Stat. Methods. 18 (1989) 3851-3874

24. E. Jones, T. Oliphant, P. Peterson. SciPy: Open source scientific tools for Python. http://www.scipy.org/, 2014.

25. R.C.P. Kerckhoffs, J.H. Omens, A.D. McCulloch. A single strain-based growth law predicts concentric and eccentric cardiac growth during pressure and volume overload. Mech. Res. Comm. 42 (2012) 40-50

26. P.L.M. Kerkhof Characterizing heart failure in the ventricular volume domain. Clin. Med. Ins. Cardiol. 9 (2015) $11-31$

27. M. Kleiber. Body size and metabolic rate. Physiol. Rev. 27 (1947) 511-541.

28. S. Klotz, I. Hay, M.L. Dickstein, G.-H. Yi, J. Wang, M.S. Maurer, D.A. Kass, D. Burkhoff. Single-beat estimation of end-diastolic pressure-volume relationship: a novel method with potential for noninvasive application. Am. J. Physiol. Circ. Physiol. 291 (2006) H403-H412

29. W. Kroon, T. Delhaas, T. Arts, P. Bovendeerd. Computational modeling of volumetric soft tissue growth: application to the cardiac left ventricle. Biomech. Model. Mechanobiol. 8 (2009) 301-309

30. Kuhl E. Growing matter - A review of growth in living systems. J. Mech. Behavior Biomed. Mat. 29 (2014) 529543

31. V. Kumar, A.K. Abbas, J.C. Aster Robbins and Cotran pathologic basis of disease, 9th edition Elsevier Saunders, 2015

32. J.D. Lee, S. Sasayama, Y. Kihara, A. Ohyagi, A. Fujisawa, Y. Yui, C. Kawai. Adaptations of the left ventricle 
to chronic volume overload induced by mitral regurgitation in conscious dogs. Heart and Vessels 1 (1985) 9-15

33. L.C. Lee, G.S. Kassab, J.M. Guccione. Mathematical modeling of cardiac growth and remodeling WIREs Syst. Biol. Med. 8 (2016) 211-226

34. I.J. Legrice, P.J. Hunter, B.H. Smaill. Laminar structure of the heart: a mathematical model. Am. J. Physiol. 272 (1997) H2466-H2476

35. D. Lewandowski, D. Kurowicka, H. Joe. Generating random correlation matrices based on vines and extended onion method. J. Multivar. Anal. 100 (2009) 1989-2001

36. E. Limpert, W.A. Stahel, M. Abbt. Log-normal distribution across the sciences: Keys and clues. Bioscience 51 (2001) 341-352

37. A. Menzel. Modelling of anisotropic growth in biological tissues. Biomech. Model. Mechanobio. 3 (2005) 147-171

38. A. Menzel, E. Kuhl. Frontiers in growth and remodeling. Mech. Res. Comm. 42 (2012) 1-14

39. J.H. Omens. Stress and strain as regulators of myocardial growth. Prog. Biophys. Mol. Biol. 69 (1998) 559-572

40. L.H. Opie, P.J. Commerford, B.J. Gersh, M.A. Pfeffer. Controversies in ventricular remodelling. Lancet. 367 (2006) 356-367

41. M. Peirlinck, K.L. Sack, P. De Backer, P. Morais, P. Segers, T. Franz, M. De Beule. Kinematic boundary conditions substantially impact in silico ventricular function. Int. J. Num. Meth. Biomed. Eng. 35 (2019) e3151

42. P. Perdikaris. Gaussian processess. A hands-on tutorial. https://github.com/paraklas/GPTutorial 2017

43. M. Raissi, P. Perdikaris, G. Karniadakis. Machine learning of linear differential equations using Gaussian processes. J. Comp. Phys. 348 (2017) 683-693

44. M. Raissi, P. Perdikaris, G. Karniadakis. Numerical Gaussian processes for time-dependent and nonlinear partial differential equations. SIAM J. Sci. Comp. 40 (2018) A172-A198

45. M.K. Rausch, A. Dam, S. Göktepe, O.J. Abilez, E. Kuhl. Computational modeling of growth: Systemic and pulmonary hypertension in the heart. Biomech. Model. Mechanobio. 10 (2011) 799-811

46. M.K. Rausch, A.M. Zöllner, M. Genet, B. Baillargeon, W. Bothe, E. Kuhl. A virtual sizing tool for mitral valve annuloplasty. Int. J. Num. Meth. Biomed. Eng. 33 (2017) $\mathrm{e} 02788$

47. J.C.L. Rodrigues, A.M. Amadu, A.G. Dastidar, G.V. Szantho, S.M. Lyen, C. Godsave, L.E.K. Ratcliffe, A.E. Burchell, E.C. Hart, M.C.K. Hamilton, A.K. Nightingale, J.F.R. Paton, N.E. Manghat, C. Bucciarelli-Ducci. Comprehensive characterisation of hypertensive heart disease left ventricular phenotypes. Heart 102 (2016) 1671-1679

48. E. Rodriguez, A. Hoger, A.D. McCulloch. Stressdependent finite growth in soft elastic tissues. J. Biomech. 27 (1994) 455-467

49. R. Rodríguez-Cantano, J. Sundnes, M.E. Rognes. Uncertainty in cardiac myofiber orientation and stiffnesses dominate the variability of left ventricle deformation response Int. J. Num. Meth. Biomed. Eng. 35 (2019) e3178

50. K.L. Sack, E. Aliotta, D.B. Ennis, J.S. Choy, G.S. Kassab, J.M. Guccione, T. Franz. Construction and validation of subject-specific biventricular finite-element models of healthy and failing swine hearts from highresolution DT-MRI. Front. Physiol. 9 (2018) 539

51. P. Saez, E. Pena, M.A. Martinez, E. Kuhl. Computational modeling of hypertensive growth in the human carotid artery. Comp. Mech. 53 (2014) 1183-1196
52. F. Sahli Costabal, F.A. Concha, D.E. Hurtado, E. Kuhl The importance of mechano-electrical feedback and inertia in cardiac electromechanics. Comp. Meth. Appl. Mech. Eng. 320 (2017) 352-368

53. F. Sahli Costabal, J.S. Choy, K.L. Sack, J.M. Guccione, G. Kassab, E. Kuhl. Multiscale characterization of heart failure. Acta Biomat. 86 (2019) 66-76.

54. F. Sahli Costabal, K. Matsuno, J. Yao, P. Perdikaris, E. Kuhl. Machine learning in drug development: Characterizing the effect of 30 drugs on the QT interval using Gaussian process regression, sensitivity analysis, and uncertainty quantification. Comp. Meth. Appl. Mech. Eng. 348 (2019) 313-333.

55. J. Salvatier, T.V. Wiecki, C. Fonnesbeck. Probabilistic programming in Python using PyMC3. Peer J. Comput. Sci. 2 (2016) e55

56. H. Sandler, H.T. Dodge. Left ventricular tension and stress in man. Circ. Res. 8 (1963) 437-445

57. S. Sasayama, J.J. Ross, D. Franklin, C.M Bloor, S. Bishop, R.B. Dilley. Adaptations of the left ventricle to chronic pressure overload Circ. Res. 38 (1976) 172-178

58. O.V. Savinova, A.M. Gerdes. Myocyte changes in heart failure. Heart Fail. Clin. 8 (2012) 1-6

59. G. Sommer, A. J. Schriefl, M. Andre, M. Sacherer, C. Viertler, H. Wolinski, G. A. Holzapfel. Biomechanical properties and microstructure of human ventricular myocardium. Acta Biomat. 24 (2015) 172-192

60. A. Tsamis, A. Cheng, T.C. Nguyen, F. Langer, D.C Miller, E. Kuhl. Kinematics of cardiac growth: In vivo charactierzaion of growth tensors and strains. J. Mech. Beh. Biomed. Mat. 8 (2012) 165-177

61. N. Townsend, L. Wilson, P. Bhatnagar, K. Wickramasinghe, M. Rayner, M. Nichols. Cardiovascular disease in Europe: Epidemiological update 2016. Eur. Heart J. 37 (2016) 3232-3245

62. J. Wong, E. Kuhl. Generating fiber orientation maps in human heart models using Poisson interpolation. Comp. Meth. Biomech. Biomed. Eng. 17 (2014) 1217-1226

63. M. Yoshida, E. Sho, H. Nanjo, M. Takahashi, M. Koboyashi, K. Kawamura, M. Honma, M. Komatsu, A. Sugita, M. Yamauchi, T. Hosoi, Y. Ito, H. Matsuda. Weaving hypothesis of cardiomyocyte sarcomeres. Am. J. Path. 176 (2010) 660-678

64. K.M. Wisdom, S.L. Delp, E. Kuhl. Use it or lose it: Multiscale skeletal muscle adaptation to mechanical stimuli. Biomech. Model. Mechanobio. 14 (2015) 195-215

65. C.M. Witzenburg, J.W. Holmes. A comparison of phenomenologic growth laws for myocardial hypertrophy. J. Elast. 129 (2017) 257-281

66. A.M. Zöllner, O.J. Abilez, M. Böl, E. Kuhl. Stretching skeletal muscle. Chronic muscle lengthening through sarcomerogenesis. PLoS ONE 7 (2012) e45661. 